\title{
Abordagens generativas em planejamento urbano: experimentos de otimização para princípios de Transit Oriented Development
}

\author{
Generative approaches in urban planning: optimization experiments for Transit Oriented Development principles \\ - Fernando Lima \\ Universidade Federal de Juiz de Fora, Brasil \\ - Jose Ripper Kos \\ fernando.lima@arquitetura.ufj.br \\ Universidade Federal de Juiz de Fora, Brasil \\ josekos@ufrj.br \\ - Rodrigo Cury Paraízo \\ Universidade Federal do Rio de Janeiro, Brasil \\ rparaizo@gmail.com
}

\begin{abstract}
This article focuses on the use of computational tools to provide dynamic assessment and optimized arrangements while planning and discussing interventions in urban areas. The objective is to address the use of algorithmic systems for evaluating and generating urban morphologies guided by Transit Oriented Development (TOD) principles. TOD is an urban development model that considers geometric and measurable parameters for designing sustainable cities. It advocates the creation of compact mixed-use neighborhoods within walking distance to a variety of transportation options and amenities, seeking to result on optimized infrastructure provision and energy efficient low-carbon districts. This paper presents algorithmic experiments for proposing a rapid-transit district, by the optimization of its urban morphology and services' location, providing an accurate and efficient TOD principles-oriented modelling.
\end{abstract}

Keywords: Generative design, Transit Oriented Development, Optimization

\section{Introdução}

Métodos computacionais de design tem sido amplamente incorporados ao processo de projeto arquitetônico. Entretanto, poucas abordagens utilizam estes recursos como suporte ao projeto urbano, a fim de desenvolver masterplans flexíveis. O paradigma paramétrico aplicado ao desenho urbano constitui uma nova possibilidade para o projeto urbanístico, que se baseia no uso de padrões e regras projetuais. O objetivo deste método é permitir a elaboração de propostas flexíveis, capazes de responder a modificações diversas. Os sistemas paramétricos diferem dos sistemas tradicionais de desenho digital por manterem a capacidade de o modelo alterar-se durante todo o processo projetual e por permitirem gerar e testar grande quantidade de versões dentro de um ambiente controlado de projeto, a partir da simples mudança de valores de um parâmetro específico. Para tanto, os softwares paramétricos têm se tornado uma ferramenta importante, pois permitem a visualização do conjunto do projeto, assim como a intervenção e atualização das partes.

Paralelamente, a questão da sustentabilidade assume, atualmente, um papel central na reflexão sobre os centros urbanos contemporâneos e as dimensões de seu desenvolvimento. $\mathrm{O}$ quadro socioambiental que caracteriza as sociedades contemporâneas revela que o impacto do homem sobre o meio ambiente está se tornando cada vez mais crítico. As cidades contemporâneas, estruturadas sob a lógica do transporte baseado no automóvel e nos grandes edifícios monofuncionais, em detrimento do transporte de massa e dos espaços públicos, devem ser repensadas e rediscutidas, e o aparato tecnológico deve estar a serviço da sociedade na busca por cidades sustentáveis. Para Rogers \& Gumuchdjian (1997), a sustentabilidade das cidades deve ser um dos principais objetivos do desenvolvimento tecnológico.

Farr (2013) defende o aumento da sustentabilidade por meio da densidade urbana. Segundo esta lógica, a densidade pode permitir reduções significativas no uso per capita de recursos. Essas reduções ocorrem proporcionalmente ao aumento da densidade urbana, e a redução da produção per capita dos gases diretamente relacionados ao efeito estufa é uma estratégia essencial para que o aumento da densidade local gere um benefício global, uma vez que os gases produzidos por atividades humanas e responsáveis por mudanças climáticas não respeitam fronteiras. Há de se considerar ainda, que o transporte nas grandes cidades é um dos maiores responsáveis pelo aumento nas concentrações de gases causadores do efeito estufa. Além de contribuir para o aquecimento global, o modelo de mobilidade urbana atual, baseado predominantemente nos deslocamentos feitos por automóvel, é responsável por enormes obstáculos nos grandes centros urbanos.

Neste contexto, o Transit-Oriented Development (TOD) apresenta-se como um modelo de desenvolvimento urbano que se enquadra no conceito do Novo Urbanismo, ao 
proporcionar mais opções de habitação e transporte, além de serviços variados que podem ser acessados a pé (priorização dos pedestres e vida urbana). Segundo Dittmar \& Ohland (2004), o TOD visa a maximizar o acesso ao transporte coletivo e não-motorizado, por meio da implantação de estações (de trem ou ônibus) centralmente localizadas e rodeadas por densidade relativamente alta. De acordo com Suzuki et al. (2013) e Dittmar \& Ohland (2004), os princípios chave para a eficiência de um projeto TOD são: (a) acessibilidade ao transporte - localização de serviços, comércio, e habitação em torno de estações de trânsito; (b) walkability - a criação de redes de rua para pedestres que conectam diretamente destinos locais a uma curta distância (1,6 km, no máximo) das paradas de transporte; (c) Diversidade - mistura de usos, densidades, tipos de habitação e custos do mesmo bairro; (d) densidade articulada para transporte de massa - o incentivo de ocupação e remodelação ao longo dos corredores de trânsito dentro de bairros existentes, permitindo que o sistema funcione de forma eficiente.

Este artigo centra-se na utilização de recursos computacionais, a fim de criar masterplans dinâmicos e adaptáveis para fornecer avaliação rápida e eficiente, nas tarefas de planejamento, discussão e proposição de intervenções em áreas urbanas. Abordou-se o uso de sistemas algorítmicos para formular estratégias para projetos urbanos sustentáveis, orientadas por princípios de TOD. Mais especificamente, o presente trabalho visa investigar como princípios mensuráveis, geométricos (e, portanto, programáveis) do TOD podem ser explicitados em um ambiente algorítmico, apresentando uma prática computacional que materialize estes conceitos e possa servir como um ponto de partida para desenvolvimentos maiores. Neste contexto, este trabalho vai no sentido de uma prática de projeto urbano sustentável que surge a partir da utilização de sistemas generativos e sua capacidade de usar cálculos complexos e recursos paramétricos a fim de aprimorar as possibilidades de projeto urbano sustentável.

\section{Transit Oriented Development}

A dependência do automóvel é um dos principais desafios que os planejadores urbanos e de transporte enfrentam nas cidades contemporâneas. Ogra e Ndebele (2014) afirmam que um crescente corpo de literatura e evidências da prática, indicam que o desenvolvimento de baixa densidade e padrões espaciais fragmentados são os principais agentes através dos quais a dependência do carro se perpetuou. A este respeito, o Transit Oriented Development tornou-se um dos paradigmas de planejamento fundamentais no sentido de reverter o desenvolvimento urbano orientado pelo automóvel. (Ogra e Ndebele, 2014).

Farr (2013) apresenta o Transit-Oriented Development (empreendimento urbano voltado para o transporte público) como um modelo de desenvolvimento urbano que que se enquadra no conceito do Novo Urbanismo, uma vez que proporciona mais opções de habitação e transporte, além de serviços variados que podem ser acessados a pé (priorização dos pedestres e vida urbana). Segundo Dittmar \& Ohland (2004), o Transit-Oriented Development (TOD) visa maximizar o acesso ao transporte coletivo e não-motorizado, por meio da implantação de estações (de trem ou ônibus) centralmente localizadas e rodeadas por densidade relativamente alta.

O Transit-Oriented Development sintetiza o que Leite (2012) entende por um modelo de desenvolvimento urbano que promova altas densidades (maior que 250 habitantes por hectare) de modo qualificado, que misture funções urbanas (habitação, comércio e serviços) e que seja baseado em um eficiente sistema de mobilidade urbana que conecte núcleos adensados e estimule a caminhada (walkability) e o ciclismo. De acordo com Leite (2012, p.158) "Com isso, otimiza-se o uso das infraestruturas urbanas e promove-se maior sustentabilidade - eficiência energética, melhor uso das águas e redução da poluição". Para Farr (2013), o TOD é mais que simplesmente um projeto próximo a uma estação de transporte público, mas sim, o conceito de um bairro em torno de uma estação, permeado por empreendimentos múltiplos, usos mistos, walkability e variedade de opções de transporte.

Walkable design, ou projeto com princípios de walkability, considera os pedestres como a mais alta prioridade. Segundo Farr (2013), o conceito de walkability consiste na capacidade que um determinado bairro possui de conectar habitações e pontos de comércio e serviço por meio de distâncias que podem ser percorridas a pé, conferindo assim, maior independência do automóvel e "uma rede viária em que se possa caminhar e um desenho que permita vida urbana e opções de transporte. " (FARR, 2013, p.109). Um instrumento que permite mensurar e, portanto, simular, programar ou reprogramar a walkability de um determinado bairro é o Walk Score, um algoritmo que, segundo Carr et al. (2011) gera uma pontuação que se baseia na distância entre uma determinada unidade de habitação e os pontos de comércio e serviço mais próximos a ela. Estes pontos são classificados em 13 categorias(mercearias, cafés, restaurantes, bares, cinemas, escolas, parques, bibliotecas, livrarias, academias de ginástica, farmácias, lojas de ferragens, lojas de roupas/música), são ponderados com mesmo peso e somados, resultando em um único índice que classifica a walkability de uma determinada localidade. Por exemplo, se em relação a uma determinada localidade o serviço mais próximo em uma categoria é de 0,4 km (5 minutos de caminhada), então o número máximo de pontos é atribuído para esta localidade nesta categoria. $\mathrm{O}$ número de pontos diminui à medida que $\mathrm{a}$ distância se aproxima de 1,6 km (30 minutos de caminhada) e não são atribuídos pontos para amenidades com mais de 1,6 km de distância. Cada categoria possui o mesmo peso e os pontos são somados e normalizados para produzir uma pontuação entre 0-100. O número de serviços disponíveis e a suas distâncias para as residências nas proximidades são os principais parâmetros para promover a walkability. 


\begin{tabular}{|l|l|}
\hline \multirow{2}{*}{$90-100$} & Walker's paradise \\
\cline { 2 - 2 } & Percursos diários não demandam automóvel. \\
\hline $70-89$ & Very walkable \\
\cline { 2 - 2 } & $\begin{array}{l}\text { A maioria dos percursos podem ser vencidos } \\
\text { a pé. }\end{array}$ \\
\hline 50-69 & Somewhat walkable \\
\cline { 2 - 2 } & Alguns percursos podem ser vencidos a pé. \\
\hline 25-49 & Car-dependent \\
\cline { 2 - 2 } & $\begin{array}{l}\text { A maioria dos percursos demandam } \\
\text { automóvel. }\end{array}$ \\
\hline $0-24$ & Car-dependent \\
\cline { 2 - 2 } & $\begin{array}{l}\text { Quase todos os percursos demandam au- } \\
\text { tomóvel. }\end{array}$ \\
\hline
\end{tabular}

Tabela 1: Classificação de valores de Walkscore. Walk Score (2014).

Desenvolvimentos de baixa densidade têm influência direta no nível atual de dependência elevada do automóvel. Assim, Ogra e Ndebele (2014) apontam que as áreas urbanas devem ser concebidas de uma forma que suporte desenvolvimentos de alta densidade, complementados por uso do solo misto e investimentos em sistemas de transportes públicos. A este respeito, altas densidades estão normalmente relacionadas a menores distâncias médias de viagem para todos os modais. Esta lógica encoraja a ocupação e remodelação ao longo dos corredores de trânsito dentro dos bairros, com o objetivo de permitir que o sistema funcione de forma eficiente. De acordo com Pushkarev e Zupan (1977), existem densidades residenciais mínimas necessárias para dar suporte a diferentes modais de transporte, como a tabela abaixo apresenta:

\begin{tabular}{|c|c|c|}
\hline Modal & Serviço & $\begin{array}{c}\text { Densidade } \\
\text { residencial mínima }\end{array}$ \\
\hline Ônibus local & $\begin{array}{c}\text { "Mínimo": para- } \\
\text { das a cada 800m, } \\
20 \text { ônibus por dia }\end{array}$ & 10,0 \\
\hline Ônibus local & $\begin{array}{c}\text { "Intermediário": } \\
\text { paradas a cada } \\
800 \mathrm{~m}, 40 \text { ônibus } \\
\text { por dia }\end{array}$ & 17,5 \\
\hline Ônibus local & $\begin{array}{c}\text { "Frequente": } \\
\text { paradas a cada } \\
800 \text {, 120 ônibus } \\
\text { por dia }\end{array}$ & 37,5 \\
\hline $\begin{array}{c}\text { Ônibus expresso } \\
\text { (Acessado a pé) }\end{array}$ & $\begin{array}{c}\text { Cinco ônibus } \\
\text { durante um } \\
\text { período de pico de } \\
\text { duas horas }\end{array}$ & $\begin{array}{c}\text { Densidade padrão } \\
\text { em cobertura de } \\
5 \mathrm{~km}^{2}\end{array}$ \\
\hline
\end{tabular}

\begin{tabular}{|c|c|c|}
\hline $\begin{array}{c}\text { Ônibus expresso } \\
\text { (Acessado por } \\
\text { automóvel) }\end{array}$ & $\begin{array}{c}\text { De cinco a dez } \\
\text { ônibus durante } \\
\text { um período de } \\
\text { duas horas }\end{array}$ & $\begin{array}{c}7,5 \\
\text { Densidade padrão } \\
\text { em cobertura de } \\
50 \mathrm{~km}^{2}\end{array}$ \\
\hline Metrô leve & $\begin{array}{c}\text { Partidas a cada } \\
\text { cinco minutos ou } \\
\text { menos durante } \\
\text { horário de pico }\end{array}$ & $\begin{array}{c}22,5 \\
\text { Densidade padrão } \\
\text { para um corredor } \\
\text { de 65 a 260km² }\end{array}$ \\
\hline $\begin{array}{c}\text { Ônibus em } \\
\text { corredor }\end{array}$ & $\begin{array}{c}\text { Partidas a cada } \\
\text { cinco minutos ou } \\
\text { menos durante } \\
\text { horário de pico }\end{array}$ & $\begin{array}{c}\text { Densidade padrão } \\
\text { para um corredor } \\
\text { de 260 a 390Km2 }\end{array}$ \\
\hline Trem suburbano & 20 trens por dia & 2,5 a 5,0 \\
\hline
\end{tabular}

Tabela 2: Densidades residenciais mínimas necessárias para suporte a diferentes modais de transporte. Pushkarev e Zupan (1977)

Apesar de se apresentar como um importante modelo de desenvolvimento com vistas a cidades mais sustentáveis, ainda não existem instrumentos ou sistemas eficientes que auxiliem os atores envolvidos na discussão, proposição e/ ou avaliação de projetos baseados na lógica do TOD a serem implementados de maneira bem-sucedida (DITTMAR \& OHLAND, 2004). Entre outros aspectos, esta dificuldade reside, principalmente, no fato de um projeto baseado na lógica TOD ser dependente de múltiplas variáveis e suas interações, baseadas em desempenho e em parâmetros geométricos, algébricos e mensuráveis, como distâncias físicas, densidades variáveis de acordo com determinados parâmetros que se relacionam com entidades geométricas: pontos (posição dos lotes), curvas (corredores de transporte) e volumes (uso do solo $\mathrm{x}$ ocupação $\mathrm{x}$ altura das edificações) que são fatores articuladores e determinantes na estruturação de um bairro TOD.

Os princípios-chave para a eficiência TOD são: (a) Transit Accessibility - desenvolvimento da vizinhança em torno de centros de trânsito e proximidade das estações; (b) Walkability - redes de ruas para pedestres que conectam destinos locais a uma curta distância de trânsito; (c) Diversidade - mistura de usos, densidades, tipos de habitação e custos no mesmo distrito; (d) densidade articulada para o transporte coletivo - ocupação e remodelação ao longo dos corredores de trânsito dentro dos bairros. Neste sentido, o design TOD é dependente de múltiplas variáveis, uma vez que ele se baseia em parâmetros geométricos e mensuráveis para um melhor desempenho. Em outras palavras, é essencialmente programável, um caso ideal para a implementação lógica algorítmica.

\section{Lógica Algorítmica}

Terzidis (2006) permite entender as posturas frente as contribuições digitais no processo de projeto em duas categorias. A primeira delas seria aquela que entende o computador apenas como uma ferramenta avançada que roda programas capazes de gerar formas sofisticadas, possibilitando 
maior domínio de concepções. Nesta lógica, entende-se que, apesar do computador alterar significativamente a natureza do projeto, não é necessário ou até mesmo desejável o aprofundamento em detalhes de seus processos internos. A segunda categoria representa o entendimento que considera inevitável o aprofundamento em questões de programação e dos processos internos dos algoritmos para conferir um uso verdadeiramente criativo ao computador. Desta forma, o pensamento é o de não limitar as soluções de projeto em experiências pré-definidas, mas sim explorar potenciais infinitos, soluções alternativas e possibilidades criativas livres de limitações.

Segundo Kilkelly (2015), o pensamento algorítmico é o oposto do pensamento intuitivo, uma vez que envolve a utilização de um processo com etapas e procedimentos definidos para a resolução de um determinado problema. Nesta lógica, a ênfase está no objetivo - o problema é resolvido ou não. Programar requer pensamento algorítmico. Programar pode significar uma maneira eficiente para auxiliar a resolver problemas urbanos complexos. Terzidis (2006) define algoritmo como uma sequência finita de instruções para resolver um problema, o que reforça o entendimento de que um algoritmo não representa, necessariamente, um programa ou software. Entretanto, combinar algoritmos com a capacidade de processamento do computador permite gerenciar grande quantidade de dados, números, cálculos e interações, potencializando as possibilidades propositivas e criando novos cenários compositivos e de desempenho com metas predefinidas.

Neste contexto de possibilidades e estratégias aplicáveis ao processo de projeto, encontram-se os sistemas generativos que Mitchell (1977) apresenta enquanto mecanismos para produzir uma variedade de soluções potenciais, orientadas para a resolução de um dado problema. Celani (2011) define sistema generativo como um método indireto de projeto, no qual o projetista não se preocupa apenas com a solução de um problema em particular em um contexto específico, mas também na construção dos parâmetros que compõem este sistema e em como as soluções serão obtidas. Nesta perspectiva, objetiva-se criar mecanismos mais ou menos genéricos, que possibilitem resolver problemas semelhantes em contextos diferentes. Kolarevic (2005) define sistemas generativos de projeto como aplicações computacionais de criação que utilizam algoritmos, parametrização, simulação e técnicas de otimização de desempenho. Esses aplicativos orientam a composição e organização a partir da avaliação das diferentes soluções, por meio de técnicas de simulação e da otimização progressiva das variáveis de performance, recorrendo a algoritmos orientados aos aspectos que se pretendem melhorar. Estabelece-se, assim, uma ferramenta de projeto que, além de ter as capacidades que se atribuem aos sistemas, inclui um feedback de desempenho na sua derivação organizacional, proporcionando, deste modo, objetivo e sentido que ultrapassam o campo estético. Esta nova abordagem de projeto inverte totalmente a tradicional, ao propor ciclos iterativos de modelagem, simulação e avaliação.

Trabalhar neste paradigma significa estabelecer, primeiramente, os objetivos que determinado projeto se propõe a resolver. Em seguida, elabora-se um conjunto de regras e restrições que definem a função que se deverá obedecer e o universo de possibilidades que o sistema generativo pode explorar. Finalmente, é introduzido um conjunto de soluções iniciais que serão progressivamente otimizadas, aproximandose das metas de desempenho previamente estabelecidas.

Conforme dito anteriormente, um projeto de TOD depende de múltiplas variáveis e suas interações, baseadas em desempenho e em parâmetros geométricos, algébricos e mensuráveis, que são essencialmente programáveis, portanto, um caso adequado para implementação de lógica algorítmica. A questão deste paper reside na possibilidade de se utilizar sistemas generativos, construídos em ambiente de Linguagem de Programação Visual, para construir modelos algorítmicos que regulem, simulem e avaliem os parâmetros necessários para projetos baseados no TOD, estabelecendo assim, uma importante e mais eficiente estratégia para elaboração de projetos de bairros e cidades sustentáveis, amparados na lógica do Transit Oriented Development.

\section{Abordagens Generativas para TOD}

Oexperimento apresentadoexplora scripting eemprega modelos algorítmicos, construídos em Grasshopper, para avaliar o potencial de aplicação de recursos paramétricos para a melhoria de resultados em projetos de TOD. É importante ressaltar, que o método proposto busca a introdução de um conjunto de ferramentas computacionais para auxiliar nos processos de projeto para bairros e cidades dentro da lógica proposta pelo TOD, e não um sistema que automatize o processo. $O$ papel do projetista nesta abordagem continua a ser central, e deve ser ele (ou os atores envolvidos no processo de design) quem irá estipular metas, alimentar o sistema e considerar aspectos subjetivos e nãoprogramáveis, como dinâmicas sociais e econômicas, entre outros. Com esta ressalva, o código generativo abordou princípios TOD, e utilizou o Galápagos para a otimização, a fim de proporcionar testes sistemáticos e ajustes otimizados para um projeto de TOD.

A programação de um sistema generativo que considere princípios TOD como parâmetros, permitiu a produção de uma enorme variedade de possíveis soluções, algumas mais, outras menos adequadas. A localização de diferentes funções urbanas, o desenho de ruas e quadras, bem como a regulação de aumento da densidade de acordo com a distância até à estação, possibilitou diversas combinações e possibilidades de arranjo. A abordagem empregada baseiase em uma lógica de interação e feedback, visando um processo de projeto eficiente que permita um maior diálogo entre os diferentes atores do processo. Neste contexto, a intenção é confirmar que a lógica algorítmica contribui de forma positiva para o processo de concepção urbana 
mais eficiente, especialmente quando aplicados como uma alternativa à complexidade das cidades contemporâneas, especificamente no que diz respeito à sustentabilidade. Sendo assim, os códigos generativos abordaram os princípios TOD em uma tarefa de design conceitual de um bairro, seguindo uma sequência de passos, como segue:

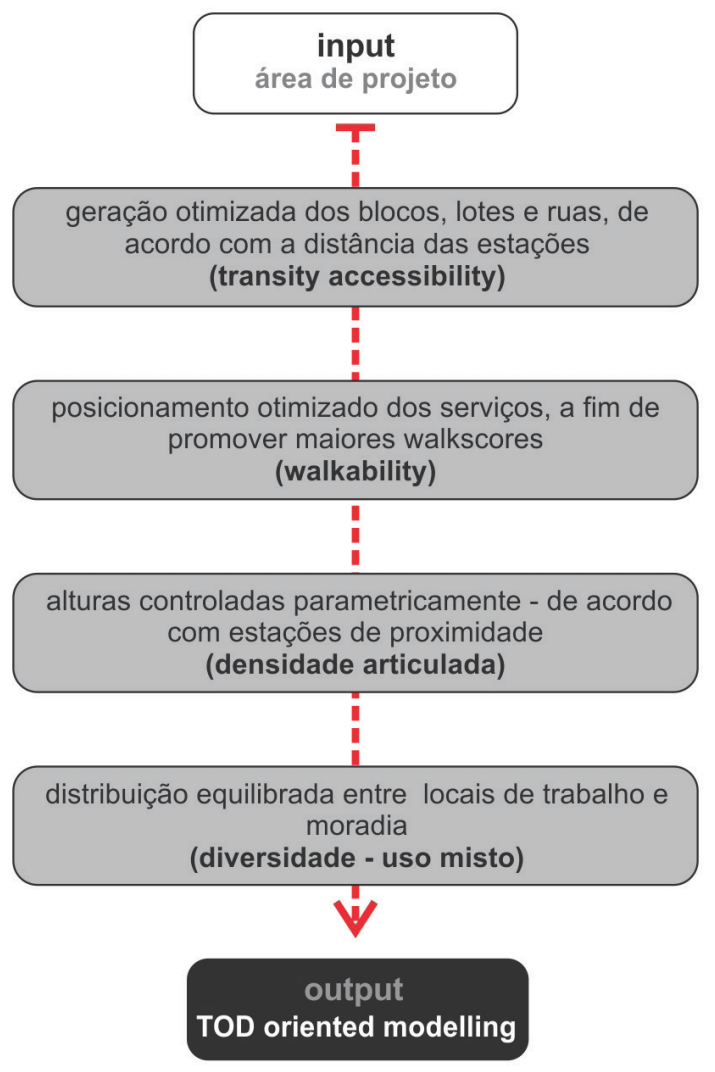

Figura 1: Lógica do algoritmo empregado.

Uma vez estabelecida a área para desenvolvimento dos experimentos, o primeiro passo foi construir uma definição algorítmica que posicionasse a estação de transporte e organizasse a rede de ruas e quarteirões. As dimensões de ruas, quadras e lotes foram definidas de acordo com princípios de TOD, em uma divisão ortogonal regular da área automatizada de sua geração. $O$ passo seguinte foi o de executar um algoritmo de optimização, a fim de encontrar as configurações mais eficientes para a geometria dos blocos e ruas, refinando automaticamente as suas dimensões e posicionamento, a fim de obter o menor deslocamento médio entre todos os terrenos e da estação. Assim, o objetivo inicial era o de garantir um bairro que iria proporcionar uma maior transit accessibility. Em outras palavras, a tarefa era descobrir quais dimensões de blocos e ruas daria a menor distância média entre todos os lotes e da estação. A ferramenta de otimização combinou milhares de possibilidades, e retornou à definição que resultou na maior transit accessibility - a menor distância média entre todos os blocos e a estação.
Após a definição do desenho ideal para lotes, quadras e ruas, o passo seguinte foi apontar quais eram as melhores posições para se inserir cada uma das cinco categorias consideradas pelo walkscore, visando fornecer a maior walkability e a possível, com o menor número de serviços. Portanto, a primeira tarefa foi a de programar uma calculadora algorítmica para estimar os walkscores globais e particulares de todos os locais dentro do perímetro de design, simulando todas as combinações possíveis de posicionamento. Estes cálculos consideraram as menores distâncias topológicas entre os locais, dada a rede de ruas proposta. Todos esses cálculos foram computadorizados e o add-on de otimização Galapagos produziu os resultados apresentados, que apontam para a localização das instalações, a fim de maximizar a média de walkscores particulares. Este procedimento foi repetido para cada uma das cinco categorias instalações, até uma disposição optimizada para cada comodidade foi encontrado. Assim, uma análise combinatória com a seleção de critérios evolutivos permitido indicar onde cada serviço estaria, de modo a proporcionar maior equilíbrio e excelentes walkscores.

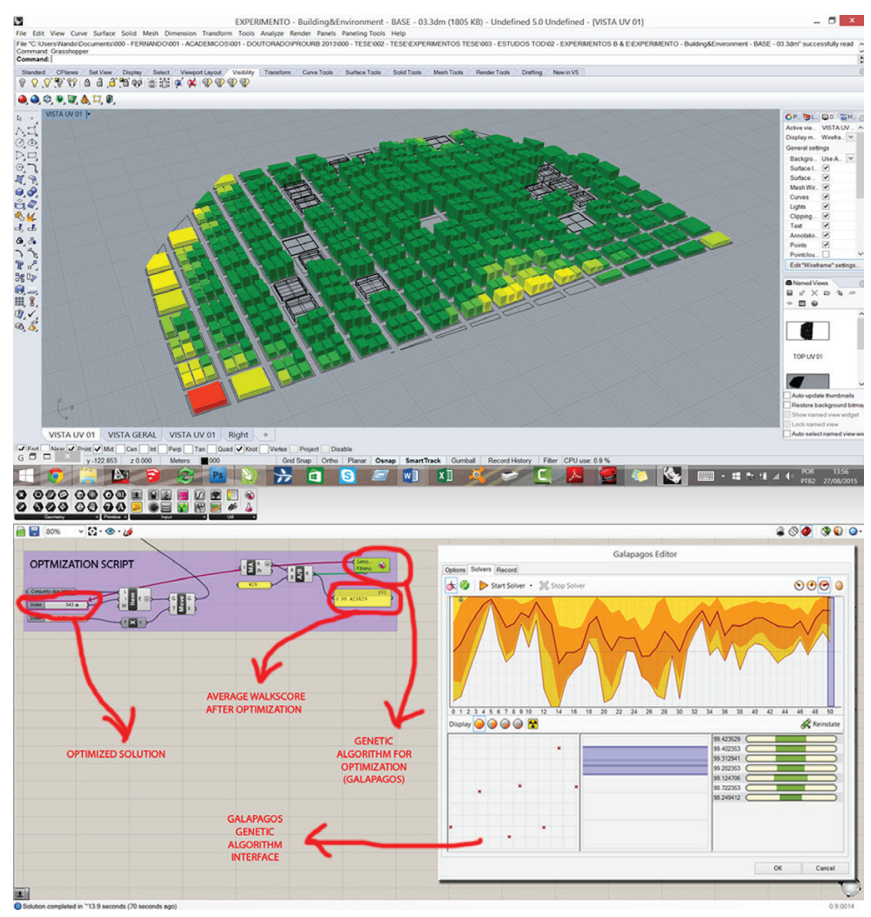

Figura 2: Modelagem algorítmica e otimização para avaliação de walkability na interface do Grasshopper.

Conforme afirmado anteriormente, a densidade é de fundamental importância para uma implementação TOD bem-sucedida. As etapas a seguir surgem da premissa que desenvolvimentos de alta densidade devem ser colocados perto de pontos de transporte, locais de trabalho e serviços, a fim de apoiar diferentes modais de transporte, como o transporte público e a caminhada. A programação paramétrica permitiu uma avaliação articulada e dinâmica das geometrias urbanas, de acordo com as densidades 
mínimas residenciais necessárias para apoiar os diferentes modais de transporte, apresentados anteriormente. Além disso, os procedimentos algorítmicos forneceram uma avaliação dinâmica do aumento da densidade, de acordo com a distância dos locais para a estação.

Operações lógicas também permitiram uma distribuição equilibrada entre pontos de trabalho e residência locais, em uma função de zoneamento capaz de assegurar uma proporção de 1/1. Esta relação especial, presente em muitas discussões TOD (Dittmar \& Ohland, 2004; Farr, 2013) é uma maneira de impor que os locais de trabalho estejam próximos as residências, e, por conseguinte, cada metro quadrado de residência no bairro corresponda um metro quadrado de área de trabalho. O metro leve foi adotado como modal primário, mas outros modais secundários foram considerados, a fim de melhorar a complexidade do modelo. A densidade crescente de acordo com a distância da estação não se apenas sobre as alturas das edificações, mas sobre o cálculo mínimo e máximo de construção que permitam satisfazer a condição fundamental que é a densidade média como um papel de apoio para os modais primários. Uma vez que um modal primário é alterado, toda a geometria da vizinhança é automaticamente rearranjada.

\section{Resultados}

O bairro proposto, algoritmicamente desenvolvido, apresenta excelente Transit Accessibility, uma vez que a distância média entre todos os terrenos e a estação é de 392,9m (menos de 5 minutos a pé), sendo a menor distância de $13 \mathrm{~m}$ (menos de 1 minuto a pé) e a maior 970m (menos de 15 minutos a pé). $\mathrm{O}$ arranjo otimizado dos serviços forneceu um walkscore médio de 99 (Walker's Paradise), com 83 (Very Walkable) como menor e 100 (Walker's Paradise) como maior pontuação. Geometrias urbanas parametricamente controladas, permitiram regular variações de densidade, de modo que ela aumente de acordo com a proximidade das estações (27-353.5 unidades habitacionais por hectare), mantendo o maior número de pessoas mais perto dos nós de transportes. No entanto, a densidade de residência global (67,7 unidades habitacionais por hectare) atende a densidade mínima residencial necessária para suportar light rail (22,5 unidades habitacionais por hectare). A diversidade também é um ponto chave para TOD. O bairro proposto possui usos múltiplos, bem como os edifícios, com as áreas térreas reservadas a locais de trabalho. A relação entre pontos de trabalho e residências locais também foi parametricamente regulada. A fim de estabelecer uma equação equilibrada, esta experiência adoptou uma proporção de 1/1, mas esta relação pode também ser modificada, rearranjando todo o sistema. A tabela a seguir, apresenta informações gerais do modelo:

\begin{tabular}{|l|c|}
\hline \multicolumn{2}{|c|}{ General information } \\
\hline Neighborhood's total area & 261.01 ha \\
\hline Neighborhood's unit 01 area & 86.99 ha \\
\hline Number of blocks & 156 \\
\hline Dimensions of "typical" blocks & $62 \times 65 \mathrm{~m}$ \\
\hline Numbers of "typical" blocks & 116 \\
\hline Streets width & $12 \mathrm{~m}$ \\
\hline Numbers of "atypical" blocks & 25 \\
\hline Number of terrains & 721 \\
\hline Number of "typical" terrains & 696 \\
\hline Dimensions of typical terrains & $19.7 \times 28 \mathrm{~m}$ \\
\hline Number of "atypical" terrains & 25 \\
\hline Housing units per hectare & 67.7 \\
\hline Compatible primary modal & Light rail \\
\hline
\end{tabular}

Transit Accessibility

Distance between terrains and station

\begin{tabular}{|c|c|c|}
\hline Lower & Higher & Average \\
\hline $13 \mathrm{~m}$ & $970 \mathrm{~m}$ & $392.9 \mathrm{~m}$ \\
\hline
\end{tabular}

\begin{tabular}{|c|c|c|}
\hline \multicolumn{3}{|c|}{ Diversity and Density } \\
\hline \multicolumn{3}{|c|}{$\begin{array}{c}\text { Density - according to station's distance } \\
\text { (housing unit per hectare) }\end{array}$} \\
\hline Lower & Higher & Average \\
\hline 27 & 353.5 & 67.7 \\
\hline Living and working places relation (un) \\
\hline Living & Working & Ratio \\
\hline 5892 & 5892 & $1 / 1$ \\
\hline
\end{tabular}

\begin{tabular}{|l|c|c|c|}
\hline \multicolumn{4}{|c|}{ Walkability } \\
\hline Partial Walkscore \\
\hline \multicolumn{1}{|c|}{ Category } & Lower & Higher & Average \\
\hline Educational & 87 & 100 & 99 \\
\hline Commercial & 77 & 100 & 99 \\
\hline Food & 82 & 100 & 99 \\
\hline Entertainment & 83 & 100 & 99 \\
\hline Recreational & 71 & 100 & 99 \\
\hline $\begin{array}{l}\text { Walkscore } \\
\text { final }\end{array}$ & 83 & 100 & 99 \\
\hline
\end{tabular}

Tabela 3: Informações gerais e os resultados obtidos na proposta. 


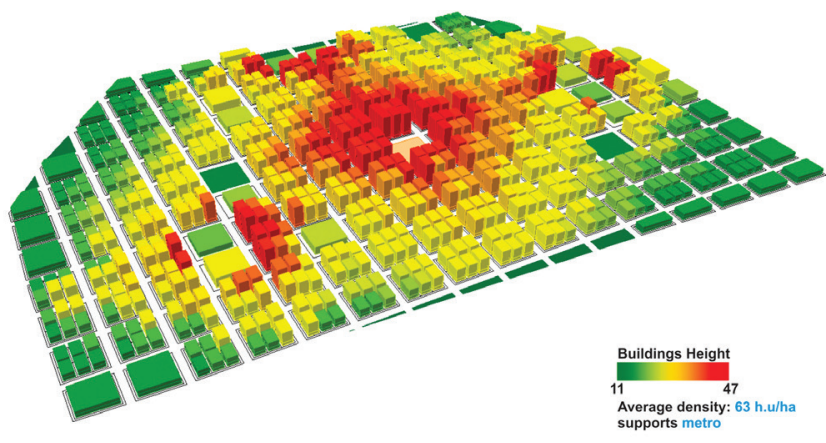

Figura 3: Densidades propostas de acordo com a distância para estação e a densidade mínima para dar suporte ao modal primário.

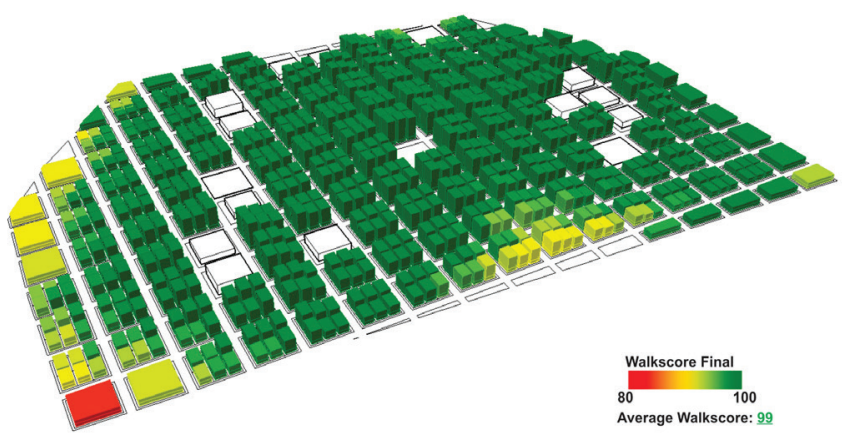

Figura 4: Mapa de cores ilustrando os walkscores obtidos no experimento.
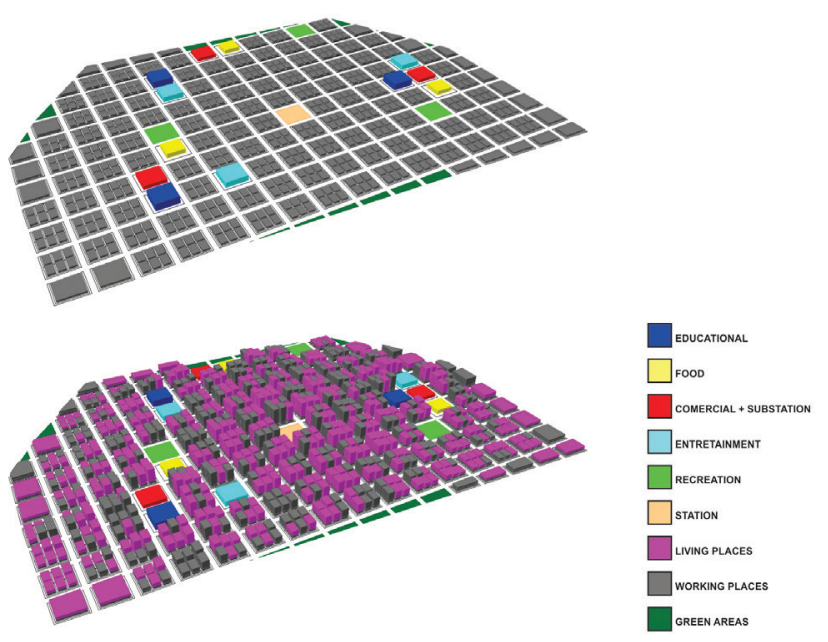

Figura 5: Usos múltiplos nos edifícios e o posicionamento dos serviços.

\section{Discussão}

Planejar cidades para uma distribuição responsável é o método mais eficaz e sustentável para reduzir o impacto do transporte urbano e promover uma vida social mais interativa nos centros urbanos. Neste contexto, a combinação de TOD e modelagem algorítmica tem o potencial para contribuir significativamente para o processo de planeamento urbano, e, até mesmo, para reduzir o aquecimento global.
O Design computacional permite gerenciar grandes quantidades de dados, em uma lógica que permite relacionar e otimizar parâmetros e disposições, de acordo com metas de desempenho pré-definidos. TOD, por sua vez, é um modelo de desenvolvimento urbano sustentável que fornece características mensuráveis e programáveis que podem ser empregados em abordagens algorítmicas em direção a arranjos mais sustentáveis para as cidades.

A forma final do modelo apresentado é o resultado da simulação de um ambiente flexível, e da avaliação e retroalimentação que lida com a relação de dados complexos. O desenho e as medidas de ruas, lotes e quadras são o resultado de um algoritmo que trabalha com base nas distâncias entre cada terreno e a estação, tendo em conta o percurso feito na rede de ruas proposta. Em outras palavras, alterar as dimensões dos blocos implica uma nova configuração de ruas e um número diferente de lotes, modificando totalmente a distância entre cada um deles. A otimização buscou a configuração que permitiu a menor distância média entre cada lote e todos os outros, para propor a configuração mais apropriada, em um procedimento que exigiu horas de cálculo, e considerou milhares de combinações.

\section{Conclusões}

A utilização de abordagens generativas proporcionou uma modelagem orientada pelo TOD precisa e eficiente, permitindo simular e avaliar vários cenários durante todo o processo de tomada de decisão. As características geométricas e mensuráveis empregadas permitiram o desenvolvimento de um sistema generativo que considera, implicitamente, os princípios TOD, resultando em um sistema que se baseia em desempenho. Este ambiente de design dinâmico permitiu que alterações em parâmetros fossem feitas de maneira interativa, proporcionando a obtenção de uma série de soluções potenciais, de maneira a procurar melhorias a cada configuração do conjunto. Ferramentas de otimização foram fundamentais para encontrar os melhores arranjos, seguindo os objetivos pré-definidos. A incorporação de um algoritmo calculador de walkscore, tornou possível promover uma excelente walkability, ajudando no cálculo de posicionamento dos serviços. Geometrias parametricamente controladas permitiram avaliar diferentes alternativas de composição, otimizando o desenho de ruas e blocos, de acordo com o posicionamento da estação. Densidade articulada, mistura de usos e equilíbrio entre lugares de trabalho e residência foram regulamentados e avaliados usando operações lógicas e regras geométricas.

Se, por um lado, TOD requer a participação de vários atores para ajudar no desenvolvimento de projetos bemsucedidos, exigindo sistemas flexíveis e eficientes, a utilização de abordagens generativas tem a capacidade de oferecer um ambiente regulatório dinâmico e preciso para apoiar os processos de tomada de decisão. Recursos de simulação, avaliação e otimização podem ser empregados 
de forma a desenvolver soluções de design otimizado. Neste contexto, a lógica computacional pode desempenhar um papel importante na tarefa de apoiar a concepção urbana sustentável, permitindo gerir e relacionar enormes quantidades de dados e suas interações, configurando uma abordagem centrada no desempenho.

\section{Agradecimentos}

Agradecemos à Fundação de Amparoà Pesquisa do Estado de Minas Gerais (FAPEMIG), ao Programa de Pós-Graduação em Urbanismo (PROURB) da Universidade Federal do Rio de Janeiro (UFRJ), bem como a Faculdade de Arquitetura e Urbanismo da Universidade Federal de Juiz de Fora (UFJF), por viabilizarem a apresentação deste artigo neste congresso.

\section{Referências}

Carr, L. B., Dunsigen, I. D., \& Marcus, B. H. (2011) Validation of walk score for estimating access to walkable amenities. British journal sports medicine, v.45, p. 1144-1158.

Celani, G. (2011). Algorithmic Sustainable Design. Uma visão crítica do projeto generativo. Resenhas Online, ano 10, n. 116.03. http://www.vitruvius.com.br/revistas/read/ drops/10.030/2109

Dittmar, H., \& Ohland, G. (2004). The new transit town: Best practices in Transit-Oriented Development. Washington: Island Press.
Farr, D. (2013). Urbanismo sustentável: Desenho urbano com a natureza. Porto Alegre: Bookman.

Leite, C. (2012). Cidades sustentáveis cidades inteligentes: Desenvolvimento sustentável num planeta urbano. Porto Alegre: Bookman.

Kilkelly, M. (2015). 5 reasons architects should learn to code. Retrieved May 7, 2015, from http://www.archdaily. com/613896/5-reasons-architects-should-learn-to-code/.

Kolarevic, B. (2005). Architecture in the digital age: design and manufacturing. Londres: Taylor \& Francis.

Mitchell, W. J. (1977). Computer-aided architectural design. Nova York: Van Nostrand Reinhold.

Ogra, A., \& Ndbele, R. (2014) The role of 6 ds in transit oriented development (TOD), in: Bahga, S. and Singla, A., Proceedings of the Neo-International Conference on Habitable Environments, CreateSpace, Jalandhar, India, 535-542.

Pushkarev, B., \& Zupan, J. M. (1977). Public transportation and land use policy. Nova York: Indiana University Press.

Rogers, R., \& Gumuchdjian, P. (1997). Cities for a small planet, Barcelona: Gustavo Gilli.

Terzidis, K. (2006). Algorithmic architecture. Nova York: Routledge.

Walk Score (2014). Walk score methodology. Retrieved from https://www.walkscore.com/methodology.shtml

Woodbury, R. (2010). Elements of parametric design. Nova York: Routledge. 